\title{
Rudimentary horn pregnancy - ten years of experience
}

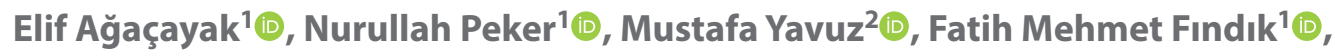 \\ Mehmet Sıddık Evsen ${ }^{1}$, , Talip Gül'10 \\ ${ }^{1}$ Department of Obstetrics and Gynecology, Dicle University, Faculty of Medicine, Diyarbakır, Turkey \\ ${ }^{2}$ Department of Obstetrics and Gynecology, Health Sciences University Diyarbakır Gazi Yaşargil Education \\ and Research Hospital, Diyarbakır, Turkey
}

\begin{abstract}
Objectives: This study aimed to evaluate data on early diagnosis and therapeutic management of rudimentary horn pregnancy (RHP).

Material and methods: Patients diagnosed with RHP at a tertiary center between for two periods of 2008-2012 and 2013-2018 were analysed retrospectively. We obtained information of patients from hospital electronic archive registration system. Data on demographic characteristics, clinical presentation, gestational age at presentation, presenting symptoms, diagnostic methods, and therapeutic management were noted and analysed by descriptive statistical method. Demographic datas, the complaint of patient's admission to hospital, history of cesarean section, preliminary diagnosis and intraoperative diagnosis were compared between periods of 2008-2012 and 2013-2018.

Results: A total of 14 RHP patients were included. Eight (57.1\%) of these patients were diagnosed between 2008-2012 (Group 1), whereas six patients (42.9\%) were diagnosed between 2013-2018 (Group 2). Rudimentary horn was non-communicating in 13 patients (92.8\%). Communicated form was observed in 1 patient in group 1. RHP was diagnosed on the left side in nine patients (64.2\%). Six of these patients were observed in group 1 and 3 were in group 2. The pre-rupture diagnosis was made in $10(71.4 \%)$ patients. Six (100\%) of 10 patients were in group 2. In addition, in group 1, four patients (50\%) experienced intraoperative RHP rupture. RHP was diagnosed before rupture in 2 (33.3\%) patients in group 2.

Conclusions: It is an indication of advanced ultrasonographic technology as well as increased carefulness on the physician side and raised alertness on the patient side that today both RHP and preoperative rupture of RHP are less frequent. Still, further awareness is required among physicians of the necessity of excision of a rudimentary horn that is detected at the time of $\mathrm{C}$-section.
\end{abstract}

Key words: Mullerian anomalies; obstetrics complications; prenatal care

Ginekologia Polska 2020; 91, 3: 117-122

\section{INTRODUCTION}

Unicornuate uterus with a rudimentary horn is an unusual Mullerian anomaly that brings about a high incidence of obstetrical complications, including ectopic pregnancy in the rudimentary horn. The actual prevalence of Mullerian duct anomalies remains unclear because of the asymptomatic state that prevails among patients; however, it is estimated to be 1:200-1:600 infertile women. Rudimentary horn pregnancy (RHP) appears in approximately $1 / 76.000$ pregnancies and usually manifests itself with firstor second-trimester uterine rupture [1]. The most frequent form of uterine anomalies is uterine septum whereas the least form is unicornuate uterus with a rudimentary horn [2]. American Society for Reproductive Medicine (ASRM) clas- sifies Mullerian anomalies into seven groups and further classifies unicornuate uterus into four sub-groups as follows: A1a) Unicornuate uterus with a communicating rudimentary horn (endometrial cavity present); A1b) Unicornuate uterus with a non-communicating rudimentary horn (endometrial cavity present); A2) Non-cavitated unicornuate uterus with rudimentary horn; B) Isolated unicornuate uterus [3]. Almost in $90 \%$ of the cases, the rudimentary horn is cavitated and non-communicating [4]. Of all RHP cases, $45-50 \%$ are asymptomatic, and only $8 \%$ of them get a clear diagnosis before symptoms become visible [5].

The purpose of this study was to evaluate data on demographic characteristics, presenting symptoms, diagnostic methods and therapeutic management of RHP for the 
periods of 2008-2012 and 2013-2018. There are several case reports and reviews in the literature on rudimentary horns. However, this is the first study in the literature to investigate and detect improvements in early diagnosis and therapeutic management of RHP, which are attributable to advanced ultrasonographic technology and increased alertness among patients.

\section{MATERIAL AND METHODS}

\section{Study Design}

The hospital records of women who diagnosed with RHP between 2008 and 2018 at the Obstetrics and Gynecology Clinic of Dicle University Hospital, which is a tertiary center public hospital were examined retrospectively. Before the study, approval was obtained from the local ethics committee of Dicle University (Approval no. 125). We searched electronic medical records from January 01, 2008 to January 01, 2018. Hospital record search regarding the keywords as the index "ectopic pregnancy", "rudimentary horn pregnancy", "horn pregnancy", "uterine horn excision", and " rudimentary horn excision" was conducted.

Ultimately, we identified 14 cases conforming to RHP (ASRM Type $2 \mathrm{~A} 1 \mathrm{a}, \mathrm{A} 1 \mathrm{~b}$ ) in this study. All patients or their parents were informed that the patients' clinical data might be used for research and scientific publications and signed an informed consent before surgery. The study period was divided into two 5-year periods. Patients diagnosed Group 1 were assigned to the pre-2013 group, whereas those diagnosed after 2012 were assigned to the Group 2. Data on demographic characteristics (age, gravidity, parity, previous history of pelvic surgery), clinical presentation (abdominal pain, missed abortus, pregnancy follow up, shock symptoms), gestational age at presentation, presenting symptoms, diagnostic method and management were analysed. The complaint of patient's admission to hospital, history of cesarean section, preliminary diagnosis and intraoperative diagnosis were compared between the two groups.

Rudimentary horns without endometrium (Type 2 A2), rudimentary horns excised and identified during surgery and unicornuate uterus without rudimentary horn (Type 2B) were excluded from the study. Also, ectopic pregnancy without rudimentary horns were excluded. Pregnancies which were determined in rudimentary horns communicant or non-communicant were included in the study. Patients were diagnosed either preoperatively with the guidance of ultrasonography (transabdominal and transvaginal) or at laparotomy. The diagnosis was established using the ultrasonographic criteria suggested by Tsafrir et al. [6]. These criteria included the following:

a) A pseudo pattern of asymmetrical bicornuate uterus,

b) Absent visual continuity tissue surrounding the gestation sac and the uterine cervix, c) Presence of myometrial tissue surrounding the gestation sac.

We used a single ultrasound device in our clinic for the pre-2013 period. We could not reach the ultrasound in the post-work. After 2012, a new technology ultrasound device was purchased in our clinic. Accessibility to ultrasonography increased.

In patients with intraoperative diagnosis, which side of the rudimentary horn is, whether the rudimentary horns with endometrium were communicated with the uterus were recorded. Patients with RHP were recorded as communicant and non-communicant according to the ASRM classification. Patients with RHP were managed by excision of the rudimentary horn combined with ipsilateral salpingectomy. All of our patients underwent laparotomy by experienced surgeons.

\section{Statistical Analysis}

Data were analysed with IBM SPSS Statistics version 21 using descriptive statistics to demographic characteristics of participants. Mean \pm standard deviation and frequency were evaluated as descriptive statistics. Chi-square test was used to evaluate categorical data. $\mathrm{p}<0.05$ was considered significant.

\section{RESULTS}

We evaluated all of the gynecologic and obstetric patients during the study period and five patients excluded from the study because of having Type $2 \mathrm{~A} 2$ and Type $2 \mathrm{~B}$ rudimentary horn. When we excluded these patients, a total of 843 ectopic pregnancies were managed in our clinic during the 10-year-period covered by this study, and RHP accounted for 14 (1.7\%) of these 843 pregnancies. Eight hundred twenty-nine cases of ectopic pregnancies other than communicated and non-communicated rudimentary uterine horn pregnancies were excluded from the study. Of the 14 patients, $8(57.1 \%)$ got a diagnosis between 2008 and 2012 (Group 1), whereas six (42.9\%) got a diagnosis between 2013 and 2018 (Group 2). Demographic data and clinical findings of each patient are shown in Table 1. The mean gestational week was $17.7 \pm 3.0$ among all of the patients. Rudimentary horn was noncommunicating in 13 patients (92.8\%) and 1 (7.2) patients communicant nature. Communicant rudimentary horn pregnancy was admitted to hospital with rupture in shock (100\%). Rudimentary horn was on the left side in 9 patients $(64.2 \%)$. All of the patients had a singleton pregnancy (Tab. 1).

There were no significant differences between groups 1 and 2 in terms of demographic data. As a preliminary diagnosis, RHP was diagnosed in 2 (33.3\%) patients in Group 2. Group 1, 6 (75\%) patients were diagnosed with EUP (p: 0.02). No patients presented with shock complaints in 


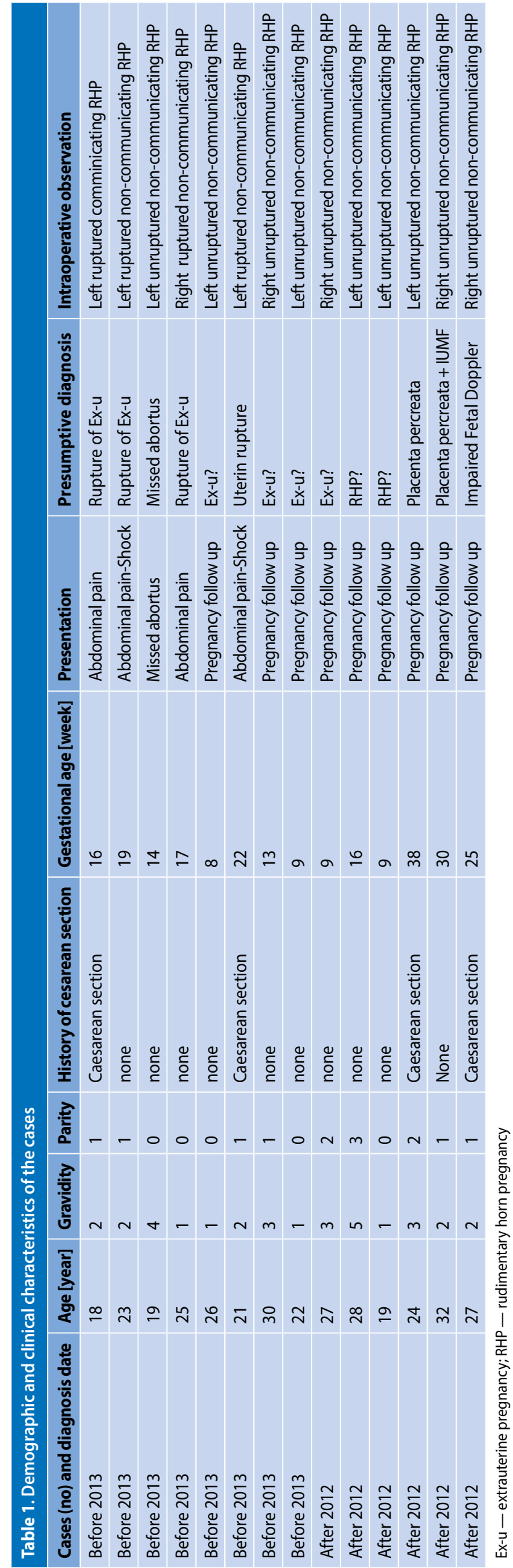

Group 2. However, there were no significant differences between the groups in terms of complaints in between two groups ( $p$ : 0.525). All patients in group 2 were diagnosed during routine pregnancy follow-up. Two patients (33.3\%) had a history of cesarean section in Group 2 (Tab. 2). Four patients (50\%) were diagnosed with intraoperative rupture Group 1 (p: 0.04). In addition, 4 patients (50\%) with a mean gestational week of $18.5 \pm 2.6$ (ranging from 16 to 22 ) experienced preoperative RHP rupture. No intraoperative rupture was diagnosed in Group 2 (Fig. 1). All of the patients underwent laparotomy for excision of the rudimentary horn.

\section{DISCUSSION}

This study provided a period-wise evaluation of data on $\mathrm{RHP}$, a rare but life-threatening condition. It demonstrated decreased frequency of both RHP and preoperative RHP rupture, increased early diagnosis of RHP with the use of preoperative ultrasonography screening, and an increased number of cases taken into surgery in a stabile state instead of a shock state in the post-2012 period compared to earlier times.

Unicornuate uterus with a non-communicating rudimentary horn stems from incomplete development of one of the Mullerian ducts with failure to fuse completely with the contralateral side [7]. Pregnancy in the rudimentary horn is an absolute emergency since pregnant rudimentary horn can rupture imminently, especially between $10^{\text {th }}$ and $15^{\text {th }}$ weeks of gestation [8]. In the present study, the mean gestational week was $17.7 \pm 3.0$ among the patients. In addition, 4 patients $(50 \%)$ with a mean gestational week of $18.5 \pm 2.6$ (ranging from 16 to 22 ) experienced preoperative RHP rupture. These patients were from the pre-2013 group, and there was no case of preoperative RHP rupture in the post-2012 group, which is attributable to advancements in ultrasonographic technology as well as an increased number of informed and careful patients who regularly attend their follow-up visits.

In a vast majority (83\%) of the patients, the rudimentary horn is non-communicating [9]. In the present study, 13 patients $(92.8 \%)$ had a non-communicating rudimentary horn.

Despite the increased use of early ultrasonography screening, it is still challenging to establish a pre-rupture diagnosis of RHP. Reports show that preoperative diagnosis is established in only $22 \%$ of gynecologic cases and $29 \%$ of obstetrical cases, which indicates that a majority of diagnoses can be confirmed only after laparotomy. Routinely performed ultrasonography, especially in the first trimester of pregnancy, assumes great importance for a clear diagnosis as well as for protection of maternal health [10]. In the present study, preliminary diagnoses made in the rest of the patients were mainly as follows: the rupture of ectopic pregnancy, uterine rupture, missed abortion and 


\begin{tabular}{|c|c|c|c|c|}
\hline & & Group 1, n: 8 [\%] & Group 2, n: 6 [\%] & $\mathbf{p}$ \\
\hline \multicolumn{2}{|l|}{ Age } & $23.0 \pm 3.9$ & $26.1 \pm 4.3$ & 0.935 \\
\hline \multicolumn{2}{|l|}{ Gravidity } & $2.0 \pm 1.0$ & $2.6 \pm 1.3$ & 0.551 \\
\hline \multicolumn{2}{|l|}{ Parity } & $0.5 \pm 0.5$ & $1.5 \pm 1.0$ & 0.089 \\
\hline \multicolumn{2}{|l|}{ Gestational week } & $14.7 \pm 4.7$ & $21.1 \pm 11.8$ & 0.010 \\
\hline \multicolumn{2}{|l|}{ History of cesarean section } & $2(25 \%)$ & $2(33.3 \%)$ & 0.733 \\
\hline \multirow{4}{*}{ Complaint of patient's admission to hospital } & Abdominal pain & $2(25 \%)$ & $1(16.7 \%)$ & \multirow{4}{*}{0.525} \\
\hline & Missed Abortus & $1(12.5 \%)$ & $1(16.7 \%)$ & \\
\hline & Shock & $2(25 \%)$ & 0 & \\
\hline & Pregnancy & $3(37.5 \%)$ & $4(66.6 \%)$ & \\
\hline \multirow{6}{*}{ Preliminary Diagnosis } & RHP & 0 & $2(33.3 \%)$ & \multirow{6}{*}{0.020} \\
\hline & EUP & $6(75 \%)$ & $1(16.7 \%)$ & \\
\hline & Uterin Ruptured & $1(12.5 \%)$ & 0 & \\
\hline & Missed Abortus & $1(12.5 \%)$ & 0 & \\
\hline & Placenta Previa & 0 & $2(33.3 \%)$ & \\
\hline & Determined Doppler & 0 & $1(16.7 \%)$ & \\
\hline \multirow{2}{*}{ Intraoperative Diagnosis } & Rupture & $4(50 \%)$ & 0 & \multirow{2}{*}{0.040} \\
\hline & Non- rupture & $4(50 \%)$ & $6(100 \%)$ & \\
\hline
\end{tabular}

Independent Student T test, $\mathrm{X}$-test $\mathrm{p}<0.05 ; \mathrm{RHP}$ - Rudimentary Horn Pregnancy; EUP — extrauterine pregnancy

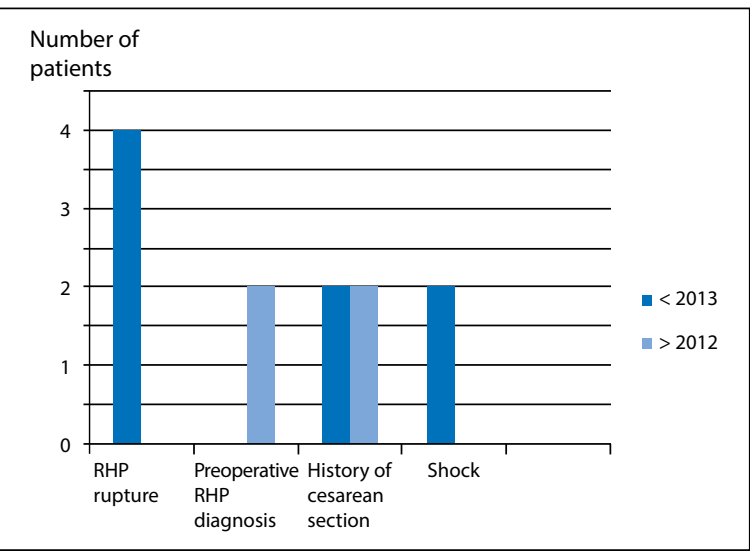

Figure 1. Clinical outcomes pre-2013 and post 2012

placenta previa. Prenatal diagnosis of RHP could be made in 2 patients (14.3\%). Those two patients that were taken into surgery with the prenatal diagnosis of RHP were both from the post-2012 group (33.3\%), which is also attributable to advancements in ultrasonographic technology.

Rudimentary horn pregnancy usually manifests itself in the form of vaginal bleeding and lower abdominal pain as seen in ectopic pregnancy. In case of rupture, patients might also experience dizziness, feebleness and collapse [11, 12]. In the present study, among of patients; 7 patients (50\%) presented with no symptoms to regular pregnancy follow-up visit whereas 3 patients (21.4\%) presented with abdominal pain, 2 patients (14.3\%) presented in a shock state and
2 patients ( $14.3 \%)$ presented with vaginal bleeding. Two patients in a shock state presented in Group 1. In Group 2, all of 6 patients were diagnosed by asymptomatically during routine pregnancy follow-up. There were no significant differences between the pre-2013 and post-2012 period.

Another cause of abdominal pain in RHP is endometriosis. Previous studies reported cases of the non-communicating rudimentary horn of unicornuate uterus with concomitant stage III pelvic and extra-pelvic endometriosis. Endometriosis reported in these cases provides support for the retrograde menstruation theory. Retrograde menstruation from ipsilateral tuba results in the development of endometriosis, which is usually severe and may cause dysmenorrhea, chronic pelvic pain, and dyspareunia [13]. None of the patients included in the present study was found to have endometriosis intraoperatively. However, three patients had complaints of abdominal pain, which might be associated with RHP rupture. Therefore, abdominal pain should be considered in pregnant women and further investigations should be performed.

Preoperative rupture, which is one of the most serious complications in rudimentary horn pregnancies, can shock the patient and endanger his life [14]. Two of our patients (14.3\%) were admitted to the hospital with shock. These patients were admitted before 2013, and although one patient had a previous cesarean section, horn excision was not performed. In this study, one of our aims is to inform the specialist physicians about the necessity of excising rudimentary horns detected during cesarean section. 
Studies in the literature showed that rupture was significantly higher in communicant rudimentary horn pregnancies. Rupture was observed in communicant $60 \%$ and non-communicants $17.6 \%$ [15]. In our study, one patient with communicating rudimentary horn pregnancy was admitted to our clinic with rupture (100\%). Rupture was observed in three of the 13 cases in non-communicant rudimentary horn pregnancy (23.1\%). We should be more careful in terms of rupture, especially in communicant rudimentary horn pregnancies.

Given the fact that myometrial tissue is thin in rudimentary horn, cases of pregnant rudimentary horn have a high frequency of uterine rupture. Therefore, its excision is recommended in the case of prophylactic purposes once it is detected intraoperatively during search for some other potential problems [16]. In the present study, 4 patients (28.5\%) had a history of cesarean section with no simultaneous excision of the rudimentary horn. Two of these four patients (25\%) were from the pre-2013 group whereas the remaining patient was from the post-2012 group, with no significant numeric differences between the two groups. In respect, we came to the understanding that physicians must be fully aware of the necessity of excision of a rudimentary horn detected at the time of cesarean section.

Pregnancy in a non-communicating rudimentary horn is believed to develop from transperitoneal sperm migration. This is a rare condition but may result in life-threating complications such as rupture, intra-abdominal bleeding and hemorrhagic shock [17]. In the present study, 2 patients (14.3\%) presented to our clinic in a shock state. Both of these patients belonged to the pre-2013 group. In the post2012 group, there were no cases of rupture or shock, which points out to the increased alertness of the importance of routine pregnancy follow-up on the patient side as well as increased use of detailed ultrasonography especially in the first trimester on the physician side after 2012. In a case report submitted in 2019, it was misdiagnosed as missed abortion and induction was tried with misoprostol that was unsuccessful. When no response to misoprostol was obtained, the diagnosis was made by ultrasonography repeat before rupture [18]. In the present study, preliminarily diagnosed as RHP were diagnosed in 2 (25\%) of 8 RHP patients from 2008 to 2012 and 2 (33.3\%) of 6 RHP patients after 2012 , with no significant numeric differences between the two groups. In this respect, it might be advanced technology and increased carefulness and precision among physicians that led to a decreased number of cases after 2012.

Following the confirmation of RHP and excision of the horn, termination of pregnancy must be considered and performed, if necessary [15]. In the present study, 2 (33.3\%) of 6 patients got a diagnosis of RHP during routine pregnancy follow-up visits after 2012. Once the RHP was confirmed, horn excision was performed in these patients despite the fact that they had no complaints. However, this study had the limitation that small sample size. When we look at the literature, studies about rudimentary horn pregnancies are generally presented as case reports. However, we think the strength of our study is one of the largest series of studies about rudimentary horn pregnancies in the literature. Besides, multi-centre studies are needed for statistical comparisons in this area.

The present study provided a period-wise evaluation of data on RHP, a rare but life-threatening condition. It resulted in the following finding: both RHP and preoperative RHP rupture were less frequent, more cases got an early diagnosis of RHP with the use of preoperative ultrasonography screening, and more cases were taken into surgery in a stabile state instead of a shock state in the period covering 2013-2018, which mainly points out to advanced ultrasonographic technology as well as increased carefulness and precision on the physician side and raised alertness on the patient side after 2012. However, further awareness is required among physicians of the necessity of excision of a rudimentary horn that is detected at the time of C-section.

\section{CONCLUSIONS}

Rudimentary horn pregnancy is a rare condition that may be misdiagnosed before surgery, have a higher risk of potential complications. Ultrasound in the first trimester may provide a means of an early diagnosis. However, the gynaecologist can be challenged for rudimentary horn diagnosis despite ultrasound. Still, further awareness is required among physicians of the necessity of excision of a rudimentary horn that is detected at the time of $\mathrm{C}$-section.

\section{REFERENCES}

1. Nahum GG. Rudimentary uterine horn pregnancy. A case report on surviving twins delivered eight days apart. J Reprod Med. 1997; 42(8): 525-532, indexed in Pubmed: 9284017.

2. Brady PC, Molina RL, Muto MG, et al. Diagnosis and management of a heterotopic pregnancy and ruptured rudimentary uterine horn. Fertil Res Pract. 2018; 4: 6, doi: 10.1186/s40738-018-0051-7, indexed in Pubmed: 30279994.

3. The American Fertility Society classifications of adnexal adhesions, distal tubal occlusion, tubal occlusion secondary to tubal ligation, tubal pregnancies, müllerian anomalies and intrauterine adhesions. Fertil Steril. 1988; 49(6): 944-955, doi: 10.1016/s0015-0282(16)59942-7, indexed in Pubmed: 3371491.

4. Speroff L, Glass RH, Kase NG. Clinical Gynecologic Endocrinology and Infertility. Sixth Edition. Williams \& Wilkins, Philadelphia 1999: 123-158.

5. Cheng $C$, Tang W, Zhang L, et al. Unruptured pregnancy in a noncommunicating rudimentary horn at 37 weeks with a live fetus: a case report. J Biomed Res. 2015;29(1): 83-86, doi: 10.7555/JBR.29.20130089, indexed in Pubmed: 25745480.

6. Tsafrir A, Rojansky N, Sela HY, et al. Rudimentary horn pregnancy: first-trimester prerupture sonographic diagnosis and confirmation by magnetic resonance imaging. J Ultrasound Med. 2005; 24(2): 219-223, doi: 10.7863/jum.2005.24.2.219, indexed in Pubmed: 15661954.

7. Junqueira BLP, Allen LM, Spitzer RF, et al. Müllerian duct anomalies and mimics in children and adolescents: correlative intraoperative assessment with clinical imaging. Radiographics. 2009; 29(4): 1085-1103, doi: 10.1148/rg.294085737, indexed in Pubmed: 19605658. 
8. Nahum GG. Rudimentary uterine horn pregnancy. The 20th-century worldwide experience of 588 cases. J Reprod Med. 2002; 47(2): 151-163, indexed in Pubmed: 11883355.

9. Thurber BW, Fleischer AC. Ultrasound Features of Rudimentary Horn Ectopic Pregnancies. J Ultrasound Med. 2019; 38(6): 1643-1647, doi: 10.1002/jum.14847, indexed in Pubmed: 30341954.

10. Arslan T, Bilgiç $E$, Sentürk $M B$, et al. Rudimentary uterine horn pregnancy: a mystery diagnosis. Fertil Steril. 2009; 92(6): 2037.e1-2037.e3, doi: 10.1016/j.fertnstert.2009.08.027, indexed in Pubmed: 19800060.

11. JayasingheY, Rane A, Stalewski $H$, et al. The presentation and early diagnosis of the rudimentary uterine horn. Obstet Gynecol. 2005; 105(6): 1456-1467, doi: 10.1097/01.AOG.0000161321.94364.56, indexed in Pubmed: 15932844.

12. Peker N, Tunc SY, Sak S. et. al. Retrospective Evaluation of the Treatment Approaches of Ectopic Pregnancies According to Gestational Week. Zeynep Kamil Tıp Bülteni. 2017; 48(1): 8-11.

13. Matalliotakis I, Goumenou A, Koumantakis G, et al. Pulmonary endometriosis in a patient with unicornuate uterus and noncommunicating rudimentary horn. Fertility and Sterility. 2002; 78(1): 183-185, doi: 10.1016/s0015-0282(02)03188-6.

14. Hussain A, Jawaid H, Faisal N, et al. Ruptured Rudimentary Horn Pregnancy Revealed on Emergency Laparotomy: A Case of Primigravida
Presenting in a Developing Country. Cureus. 2018; 10(5): e2591, doi: 10.7759/cureus.2591, indexed in Pubmed: 31489271.

15. Li X, Peng $P$, Liu $X$, et al. The pregnancy outcomes of patients with rudimentary uterine horn: A 30-year experience. PLoS One. 2019; 14(1): e0210788, doi: 10.1371/journal.pone.0210788, indexed in Pubmed: 30682068.

16. Sefrioui $O$, Azyez $M$, Babahabib A, et al. [Pregnancy in rudimentary uterine horn: diagnostic and therapeutic difficulties]. Gynecol Obstet Fertil. 2004; 32(4): 308-310, doi: 10.1016/j.gyobfe.2004.01.015, indexed in Pubmed: 15123100.

17. Lai YJ, Lin $\mathrm{CH}$, Hou WC, et al. Pregnancy in a noncommunicating rudimentary horn of a unicornuate uterus: Prerupture diagnosis and management. Taiwan J Obstet Gynecol. 2016; 55(4):604-606, doi: 10.1016/j. tjog.2016.06.009, indexed in Pubmed: 27590392.

18. Parveen R. Detection and Management of Pregnancy in Rudimentary Horn of Uterus. J Coll Physicians Surg Pak. 2019; 29(6): S70-S72, doi: 10.29271/jcpsp.2019.06.S70, indexed in Pubmed: 31142429.

19. Lennox G, Pantazi $S$, Keunen J, et al. Minimally invasive surgical management of a second trimester pregnancy in a rudimentary uterine horn. J Obstet Gynaecol Can. 2013; 35(5): 468-472, doi: 10.1016/S17012163(15)30938-5, indexed in Pubmed: 23756278. 